\title{
Crossover between macroscopic and mesoscopic regimes of vortex interactions in type-II superconductors
}

\author{
Rafael Zadorosny, ${ }^{1}$ Edson Sardella, ${ }^{2}$ André Luiz Malvezzi, ${ }^{3}$ Paulo Noronha Lisboa-Filho, ${ }^{3}$ and Wilson Aires Ortiz ${ }^{4,5}$ \\ ${ }^{1}$ Faculdade de Engenharia de Ilha Solteira, Universidade Estadual Paulista, Departamento de Física e Química, Caixa Postal 31, 15385-000, \\ Ilha Solteira, São Paulo, Brazil \\ ${ }^{2}$ Universidade Estadual Paulista, Instituto de Pesquisas Meteorológicas, CEP 17048-699 Bauru, São Paulo, Brazil \\ ${ }^{3}$ Universidade Estadual Paulista, Departamento de Física, Faculdade de Ciências, Caixa Postal 473, 17033-360, Bauru, São Paulo, Brazil \\ ${ }^{4}$ Departamento de Física, Universidade Federal de São Carlos, 13565-905, São Carlos, São Paulo, Brazil \\ ${ }^{5}$ Centre for Advanced Study, Norwegian Academy of Science and Letters, NO-0271, Oslo, Norway \\ (Received 1 November 2011; revised manuscript received 23 May 2012; published 11 June 2012)
}

\begin{abstract}
In the present work we report the existence of a crossover between the macroscopic and mesoscopic regimes of vortex interactions in type-II superconductors. Our findings rely on a systematic procedure to determine this crossover, which is based on the influence of the surface on the vortex structure of small superconductors. An adjacent result that we have found is that near this regime transformation, the vortex lattice develops a progressive change of symmetry, from square to hexagonal, which is intimately related to the meso-to-macro crossover. Our numerical simulations have been done for a long superconducting cylinder of square cross section for a wide range of length scales and temperatures.
\end{abstract}

DOI: 10.1103/PhysRevB.85.214511

PACS number(s): 74.25.-q, 74.20.De, 74.78.Na

\section{INTRODUCTION}

In condensed matter physics, samples are usually considered to be large enough that the influence of finite dimensions on their properties is negligible; therefore, bulk behavior is assumed. This is not the case, however, in many situations of interest. Examples range from quantum size effects in metallic nanoparticles ${ }^{1,2}$ and microfluidics, ${ }^{3}$ to cluster size effects. ${ }^{4}$ Hallmark physical ingredients in these systems are large surface-to-volume ratio and one or more dimensions of the sample approaching a relevant fundamental scale.

Mesoscopic superconductors also constitute a class of materials where size effects can play a relevant role to determine the vortex arrangement throughout the specimen. ${ }^{5-16}$ In fact, even when only a few quanta of magnetic flux are nucleated into a mesoscopic sample, vortex interactions with other vortices and with the screening currents circulating around the edges give rise to a variety of configurations, such as giant vortices, multivortex states, or even hybrid states of both types. These configurations are strongly influenced by both the geometry and size of the sample. The occurrence of giant vortices in coherence length-sized samples have been predicted by several theoretical calculations, ${ }^{17-22}$ although in some exotic confined geometries, such as a circular sector, ${ }^{23}$ they were not observed.

Several authors have tried to detect this state experimentally. Kanda et al ${ }^{9-11}$ used multiple small tunnel junction measurements. They found that, by putting several small tunnel junctions symmetrically distributed on the disk edge, for some vorticities, they could not find any significant difference in the voltages at the contacts. This is taken as an indication of the occurrence of a giant vortex state. Some other authors have attempted to observe giant vortices by direct imaging technique. By using SQUID microscopy, in Refs. 14-16 they could not find giant vortex states in mesoscopic superconducting square and triangle samples. However, on using the Bitter pattern decoration technique in a niobium superconducting disk, Grigorieva et al ${ }^{13}$ observed clusters of multivortex states coexisting with giant vortices. In another work, Cren et al. ${ }^{12}$ studied the vortex states in ultrathin single nanocrystals of $\mathrm{Pb}$ with random geometries via scanning tunneling microscopy. The images were taken under several values of applied field and at very low temperature. They showed that under certain conditions of magnetic field and sample size, a giant vortex is formed and in the vicinity of the core center the order parameter evolves as $|\psi| \propto r^{L}$ where $L$ is the vorticity.

In the present work we show strong evidence that there exists a region of crossover between these vortex states in mesoscopic superconductors and the Abrikosov hexagonal vortex lattice in macroscopic superconductors. We have done so by studying the influence of the surface on the vortex configuration as a function of the sample size and temperature. Based on the physics governing the crossover between the mesoscopic and macroscopic behavior, we developed a criterion to determine a length scale that delineates the frontier between those two regimes. One could speculate that this crossover would occur for sample sizes of the order of the penetration depth $\lambda(T)$. As our results will show later on, the length scale that delimits the mesoscopic-macroscopic frontier differs quite significantly from what one might suspect.

The problem treated here was investigated within the scope of the Ginzburg-Landau theory. It is not uncommon to find papers arguing whether the Ginzburg-Landau theory could be applicable or not to the study of superconductivity at a mesoscopic level. This relevant issue has been discussed in detail in Refs. 17,24, and 25. In the latter, the authors have made a comparative analysis between the phenomenological Ginzburg-Landau theory and the microscopic Bogolyubov-De Gennes theory, for a mesoscopic superconducting square. They conclude that both theories produce very similar results.

The outline of this paper is as follows. First we provide a brief overview of the theoretical model used to obtain the equilibrium configurations of the superconducting state. Then, we describe the numerical method employed to solve the time-dependent Ginzbug-Landau (TDGL) equations for a superconductor in the presence of an external applied magnetic 
field. The remainder of the paper is devoted to presentation and discussion of the results that emerge from our numerical simulations.

\section{THEORETICAL FORMALISM}

The superconducting state is usually described by the complex order parameter $\psi$. The quantity $|\psi|^{2}$ represents the electronic density of Cooper pairs. In the regions where $|\psi|^{2}$ is small, superconductivity is suppressed. At the center of the vortex $|\psi|^{2}=0$, whereas the local magnetic field $\mathbf{h}$ is maximum. The order parameter and the local magnetic field can be determined by the Ginzburg-Landau equations which, in their time-dependent formalism, are expressed by ${ }^{26}$

$$
\begin{aligned}
\left(\frac{\partial}{\partial t}+i \Phi\right) \psi & =-(-i \nabla-\mathbf{A})^{2} \psi+(1-T) \psi\left(1-|\psi|^{2}\right), \\
\beta\left(\frac{\partial \mathbf{A}}{\partial t}+\nabla \Phi\right) & =\mathbf{J}_{s}-\kappa^{2} \nabla \times \mathbf{h},
\end{aligned}
$$

where $\mathbf{J}_{s}=(1-T) \Re\left[\psi^{*}(-i \nabla-\mathbf{A}) \psi\right]$ is the supercurrent density; $\psi$ is the order parameter, $\mathbf{A}$ is the vector potential, related to the local magnetic field as $\mathbf{h}=\boldsymbol{\nabla} \times \mathbf{A}$, and $\Phi$ is the scalar potential. Here, the distances are measured in units of the coherence length at zero temperature $\xi(0)$; the magnetic field is in units of the zero temperature upper critical field $H_{c 2}(0)$; the temperature $T$ is in units of the critical temperature $T_{c}$; the time is in units of the characteristic time $t_{0}=\pi \hbar / 8 k_{B} T_{c} ; \kappa$ is the Ginzburg-Landau parameter; $\beta$ is the relaxation time of $\mathbf{A}$, related to the conductivity. We have adopted a linear dependence with respect to temperature for the phenomenological parameters in the Ginzburg-Landau theory [i.e., $H_{c 2}(T)=H_{c 2}(0)(1-T)$ ]. For small size superconductors this is also valid for temperatures well below $T_{c}$, despite the microscopic derivation of the TDGL equations being valid only for $T$ very close to $T_{c} \cdot{ }^{27}$ Notice that the TDGL equations and their discretized form ${ }^{21,28}$ are gauge invariant under the transformations $\psi^{\prime}=\psi e^{i \chi}, \mathbf{A}^{\prime}=\mathbf{A}+\nabla \chi, \Phi^{\prime}=$ $\Phi-\partial \chi / \partial t$. We chose the zero-scalar potential gauge, that is, $\Phi=0$ at all times and positions.

In order to solve the TDGL equations, we have used the link variables method as delineated in Ref. 28. There are several extensions of this method. For instance, in Ref. 29 it has been adapted for very thin superconductors and in Ref. 23 for circular geometries. The simulations were carried out for samples with square geometry submitted to external magnetic fields applied along the cylinder axis, which is considered infinite. In principle, the TDGL equations can provide all transient states for a fixed external applied magnetic field. However, in this work we are only interested in the stationary states. We solved these equations starting from zero applied field, which was then increased adiabatically in small steps, $\Delta H=10^{-4}$, until superconductivity was completely destroyed. At each step we let the system attain its equilibrium configuration and use such state as the initial condition for the next applied field.

\section{RESULTS AND DISCUSSION}

Let us now turn to the presentation of the results that arise from the numerical solution of equations (1). Our simulations have been carried out by using $\beta=1$ and $\kappa=5$ for a large number of superconducting square samples of side $L$, given in units of $\xi(0)$. Since we are interested only in the stationary states, this choice of $\beta$ is adequate for the purposes. The lateral dimensions of the sample were varied in steps of $\Delta L=1$ [also expressed in units of $\xi(0)]$ and the temperature of simulations ranged from $T=0$ to $T=0.9375$ (in units of $T_{c}$ ).

The crucial issue here is to define a robust criterion to determine the characteristic size regulating the threshold among mesoscopic and macroscopic behavior. The following discussion depicts the reasoning that led us to stipulate this norm. It is well known that vortices nucleate at the central portions of the surface (i.e., not at the corners). Their complete development is preceded by a progressive deformation of the screening current mainstream which, otherwise, while the sample is in the Meissner phase, is parallel to the faces. As the applied field $H$ is raised, this deformation proliferates into the sample until a vortex is completed. At this point, what was just a twist at the inception of the penetration process, becomes a curled screening current, enclosing the ingoing vortex. Thus, the pursuit for the threshold of the genuine mesoscopic behavior is equivalent to the search for the range of the interaction among vortices, which are just nucleating at the surfaces. Since this interaction is mediated by the superconducting regions, it should be detectable through all relevant superconducting properties (e.g., the order parameter, the magnitude and shape of the screening currents, and even the sample magnetization $M$ ).

Figure 1 illustrates this correlation for two samples of different sizes. As will become clear throughout this discussion, the largest among them $(L=76)$ has a macroscopic behavior, while the other $(L=26)$ exhibits mesoscopic superconductivity. The magnetization versus field curves shown in Fig. 1(a) for both samples exhibit clear jumps at the field $H_{j}$, corresponding to the first entrance of vortices into the sample. Noticeably, $M(H)$ for sample $L=26$ develops a local minimum at $H_{m}$, nonexistent for sample $L=76$. This minimum reflects a delay (in field) of vortex invasion, due to the repulsive interaction between the entities, which are nucleating at the borders [see Fig. 1(b), upper $L=26$ panel]. Differently from the case for the larger sample [Fig. 1(b), upper $L=76$ panel], the excessive proximity of the surfaces in sample $L=26$ causes a strong repulsion between each vortex and its counterparts, even while embryonic at the other surfaces, which causes a nonnegligible degradation on the screening capability, due to the extra repulsive barrier. This degradation is clearly manifested on $M(H)$ curves, whose deviation from the Meissner response for a bulk sample $(-4 \pi M=H)$ increases with decreasing $L$. The upper $L=26$ and $L=76$ panels in Fig. 1(b) also capture the situation, delineating the order parameter at the field $H_{j}$. The color code is such that dark red regions are fully screened, while dark blue tones represent strongly suppressed superconductivity. While four vortices ${ }^{30}$ are being generated at the surfaces of both samples, the order parameter at the very center of the macroscopic sample $(L=76)$ is preserved, even though vortices are just about to enter; contrarily, the tones 


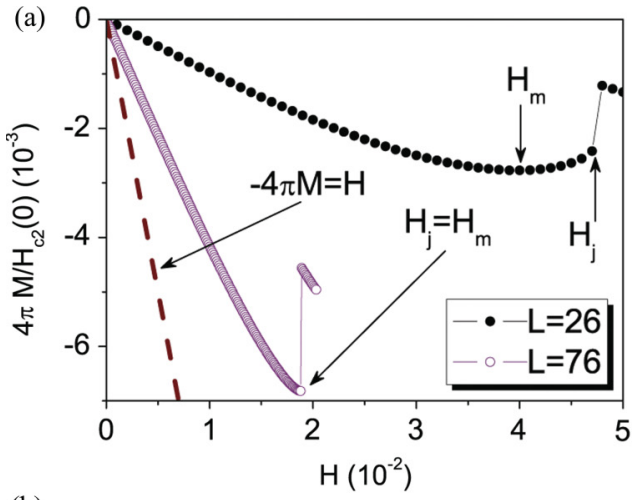

(b)

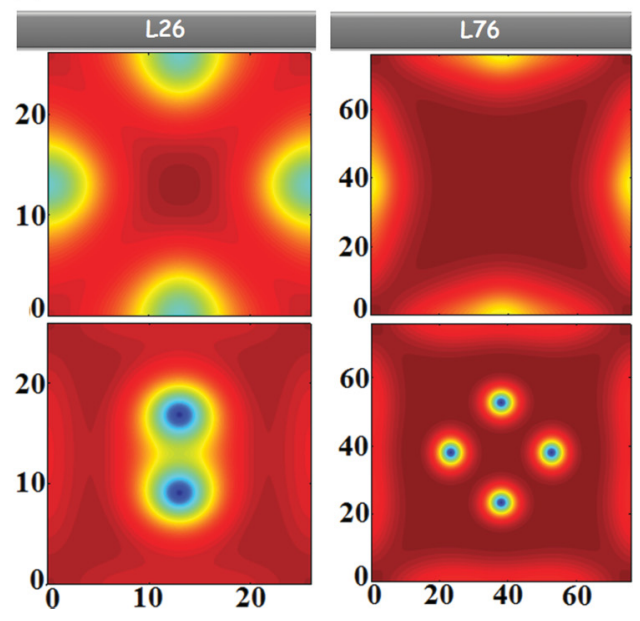

FIG. 1. (Color online) (a) Magnetization versus field curves for samples $L=26$ and $L=76$ at $T=0.875$. As $L$ increases, $H_{j}$ and $H_{m}$ (seen for $L=26$ ) tend to approach each other and the minimum on the magnetization curve, coincident with $H_{j}$ for $L=$ 76 , disappears for $L>76$. on the magnetization curve disappears. (b) Upper and lower $L=26$ panels show the order parameter for this sample, immediately before and after the first entrance of vortices ( $H_{j}$ and $H_{j}+10^{-4}$, respectively). The same applies for the upper and lower $L=76$ panels. Noticeably, as discussed in the text, only two vortices appear for $L=26$. Notice also that the sample sizes are represented as equal, although their side ratio is $76 / 26 \approx 3$.

at the central portion of the mesoscopic sample $(L=26)$ are somewhat lighter, indicating that screening currents are already circulating in that region, and the nucleating vortices do interact with each other. The lower $L=26$ and $L=76$ panels in Fig. 1(b) depict the situation immediately after vortex entrance (i.e., at $H=H_{j}+10^{-4}$ ) further emphasizing the repulsion among the entities that are being formed. While for the larger sample, all nucleating vortices manage to enter at $H_{j}$, the mesoscopic sample is not able to host all four at once and, as they would not fit along, only two vortices enter.

Even though one can visualize such differences and, accordingly, infer the existence of a limiting frontier among both regimes, it is not an easy task to assign a specific value of $L$ for the threshold size. As will be further discussed in this paper, the meso-to-macroscopic frontier separates a region of pure macroscopic behavior from another where mesoscopic and macroscopic characteristics coexist. Based on our simulations for a large number of samples with different sizes, we came

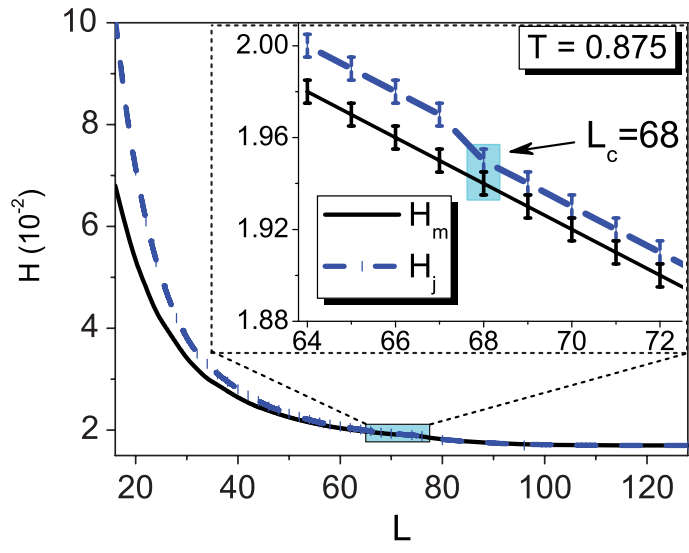

FIG. 2. (Color online) Size dependence of $H_{j}$ and $H_{m}$, indicating that both superimpose (within the precision bar of the simulations) above the threshold value $L_{c}$. The zoomed area highlights the saturation point.

to the conclusion that the occurrence of local minima on the magnetization curves is due solely to the additional repulsion among vortices, which become important when the sample dimensions are sufficiently small, a feature that we take as the guiding line to quantify the meso-macro threshold criterion for such a rather subtle crossover. Thus, for a fixed temperature, we take the critical size $L_{c}$ as that for which $H_{m}=H_{j}$. This means that, for sizes greater than $L_{c}$, the sample behaves as a macroscopic superconductor, in the sense that vortices nucleating at opposite sides do not interact before entering; below $L_{c}$, the mesoscopic regime dominates, as a consequence of the size of the sample being smaller than the interaction range. Figure 2 shows the size dependence of $H_{m}$ and $H_{j}$ for $T=0.875$. The zoomed up box emphasizes the difference among both quantities; the vertical bars correspond to the width of the field step in our simulations, $\Delta H=10^{-4}$. Taking the critical size as that above which the vertical bars start to overlap, we determined $L_{c}=68$ for this temperature.

We have also investigated the surface contribution to the magnetic free energy of the system in the whole range of values of $L$ studied here. At the jump, this energy term is proportional to the difference among two field integrals, between zero and $H_{j}$, of the magnetization: the bulk (i.e., surface-free) case, exhibiting a perfect Meissner response $(-4 \pi M=H)$, and the finite sample, for which the magnetization is affected by repulsion among nucleating vortices. To illustrate this point, the energy of the state of the sample immediately before the first penetration is shown in Fig. 3, as a function of the sample size. As can be seen, such energy decreases monotonically with $L$ and its derivative saturates, within a certain precision indicated by the error bars, at a length scale that is quite close to $L_{c}(T)$ found by the criterion described previously. Our search for other evidences of this regime transformation included the size dependence of the initial slope of $M(H)$, which also exhibits a smooth variation with respect to $L$.

The following remarkable characteristics of this meso-tomacroscopic crossover were observed: (i) for $L>L_{c}$, at any temperature, the symmetry of the vortex lattice is hexagonal, as shown in the upper $L=96$ panel of Fig. 4, which also indicates that it is destroyed upon increase of the field, from $H=0.126$ 


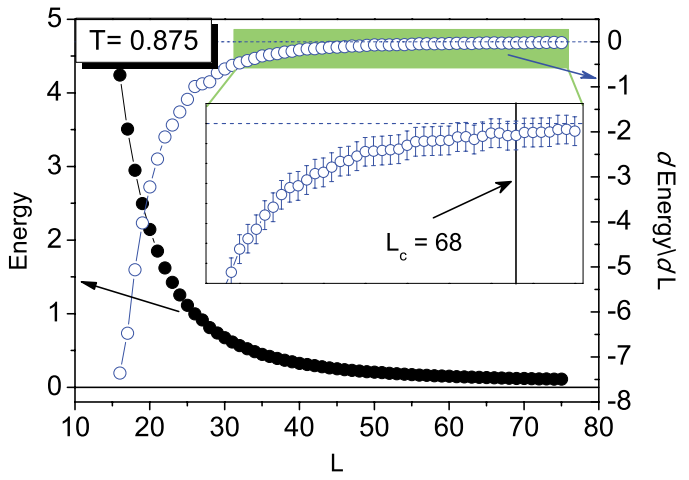

FIG. 3. (Color online) Black circles: energy as a function of $L$; blue circles: derivative of the energy with respect to $L$. The energy was calculated as $\int_{0}^{H_{j}} B d H$, where $B$ is the induction, and $H_{j}$ is the field right before the first entrance of vortices; in this calculation, only the magnetic field energy inside the superconductor is accounted for. The temperature is $T=0.875$.

to $H=0.127$, and that superconductivity remains only at the borders. Notice that there is a quite close coincidence between this field and $H_{c 2}(T=0.875)=1-0.875=0.125$, which represents the applied field sufficient to destroy the vortex lattice for a bulk superconductor. We will comment further about it later on. (ii) For $L \ll L_{c}$ the vortex lattice most predominantly has square symmetry; depending on $T$ and $L$, one can also have giant vortex states coexisting with single vortices. $^{20,21}$ Such behavior can be seen in the lower $L=20$ panel of Fig. 4; for $H=0.110$, two vortices penetrate the sample and a giant vortex with two flux quanta is formed at the center. Nonetheless, for $H=0.126$, two more vortices enter into the sample and the giant vortex splits in four separate entities, following the fourfold symmetry of the problem. (iii) The most interesting characteristic of the meso-to-macro occurs for $L \lesssim L_{c}$; for high external applied fields, that is, close to the Abrikosov upper critical field $H_{c 2}(T)$, when the density of vortices is large, the lattice develops a progressive crossover from hexagonal to square symmetry. We illustrate this scenario in the $L=64$ panels of Fig. 4 for $T=0.875$. As shown in this figure, for applied fields still low but sufficient to form a vortex lattice ( $H=0.110)$ the symmetry is hexagonal. As we increase the field, the symmetry is somewhat mixed, with hexagons at the central portions of the sample and squares closer to the edges, as illustrated for $H=0.113$ and $H=0.123$. For even higher fields $(H=0.127)$, the lattice is eventually destroyed and surface superconductivity survives only at the surface.

We have also studied the system for lower temperatures, as exemplified in Fig. 5 for $T=0.3125$, at which $L_{c}=$ 38. To describe the vortex configurations around $L_{c}$, two samples were chosen, one right below $(L=36)$ and the other right above $(L=40)$ the crossover value. For $H=0.600$, vortices are displayed in hexagonal lattices for both samples. However, at $H=0.620$ and $H=0.694$, triangular and square vortex lattices coexist for sample $L=36$, whereas for these same values of the applied field, the vortex lattice remains triangular for sample $L=40$. Eventually, at sufficiently high applied fields, the vortex lattice is entirely destroyed on both samples; for $T=0.3125$ the suppression of the vortex
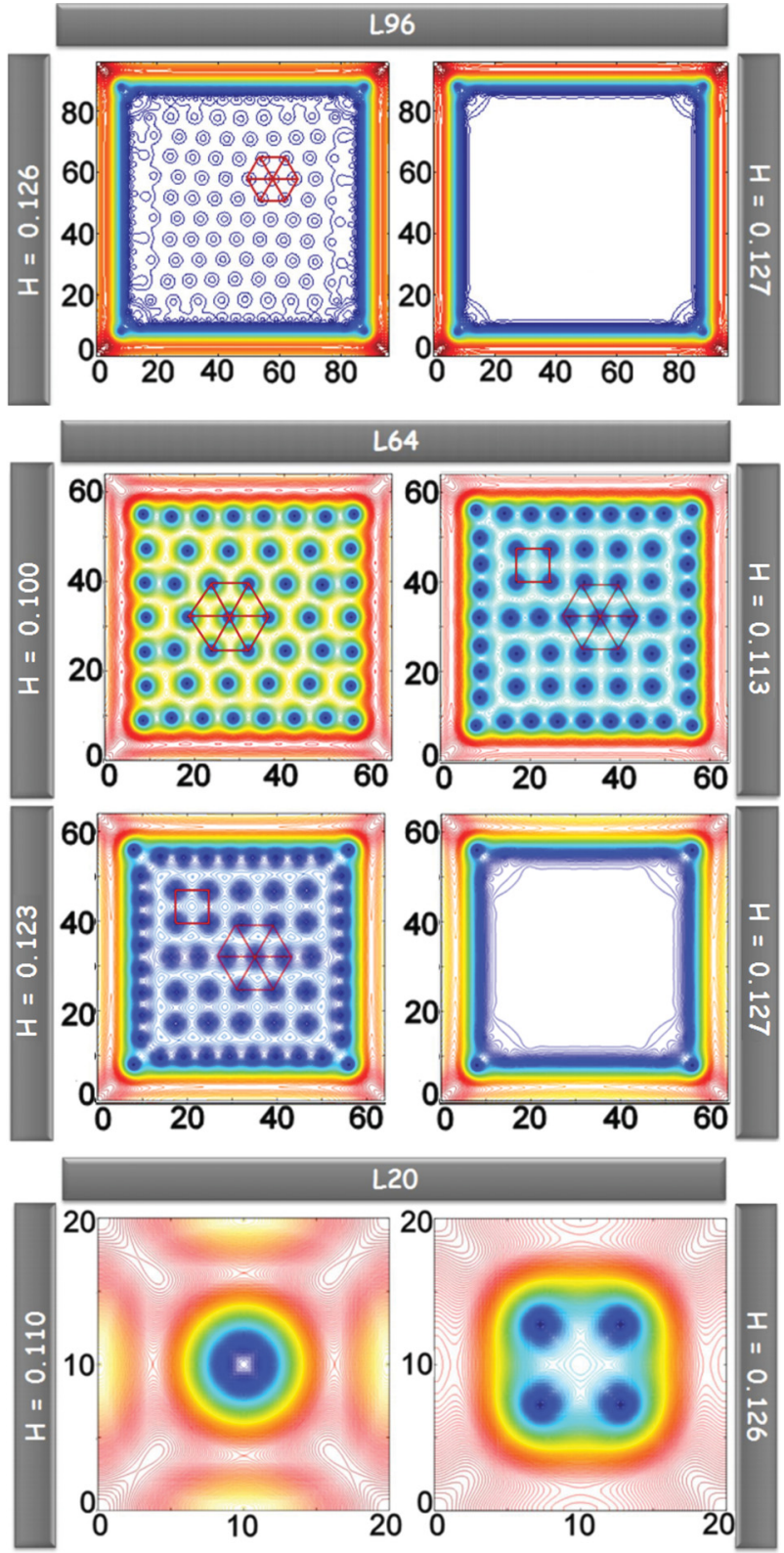

FIG. 4. (Color online) Vortex lattice for samples $L=96, L=64$, and $L=20$ for several values of the external field at temperature $T=0.8750$. The upper panel shows that a hexagonal vortex lattice is maintained until the suppression of superconductivity in the center of the sample $L=96$. In the middle panel, the crossover of the vortex lattice, that takes place below the threshold line, is shown for sample $L=64$. The lower panel shows that, far below the threshold line $L_{c}$ (in this case $L=20$ ), the vortices follow the symmetry of the sample due to size effects.

lattice occurs at $H=0.695$. Again, notice that this field, as already seen for $T=0.875$, is very close to the upper critical field $H_{c 2}(T=0.3125)=1-0.3125=0.6875$. We see thus that, for different temperatures, the value of the applied field sufficient to destroy the lattice, but not superconductivity at the surface, is quite close to the Abrikosov field $H_{c 2}(T)$ for bulk samples. We take this as a robust evidence of the accuracy of our simulations. At $T=0.875$, only surface superconductivity remains on sample $L=64$ for $H \geqslant 0.127$ (Fig. 4); the same 


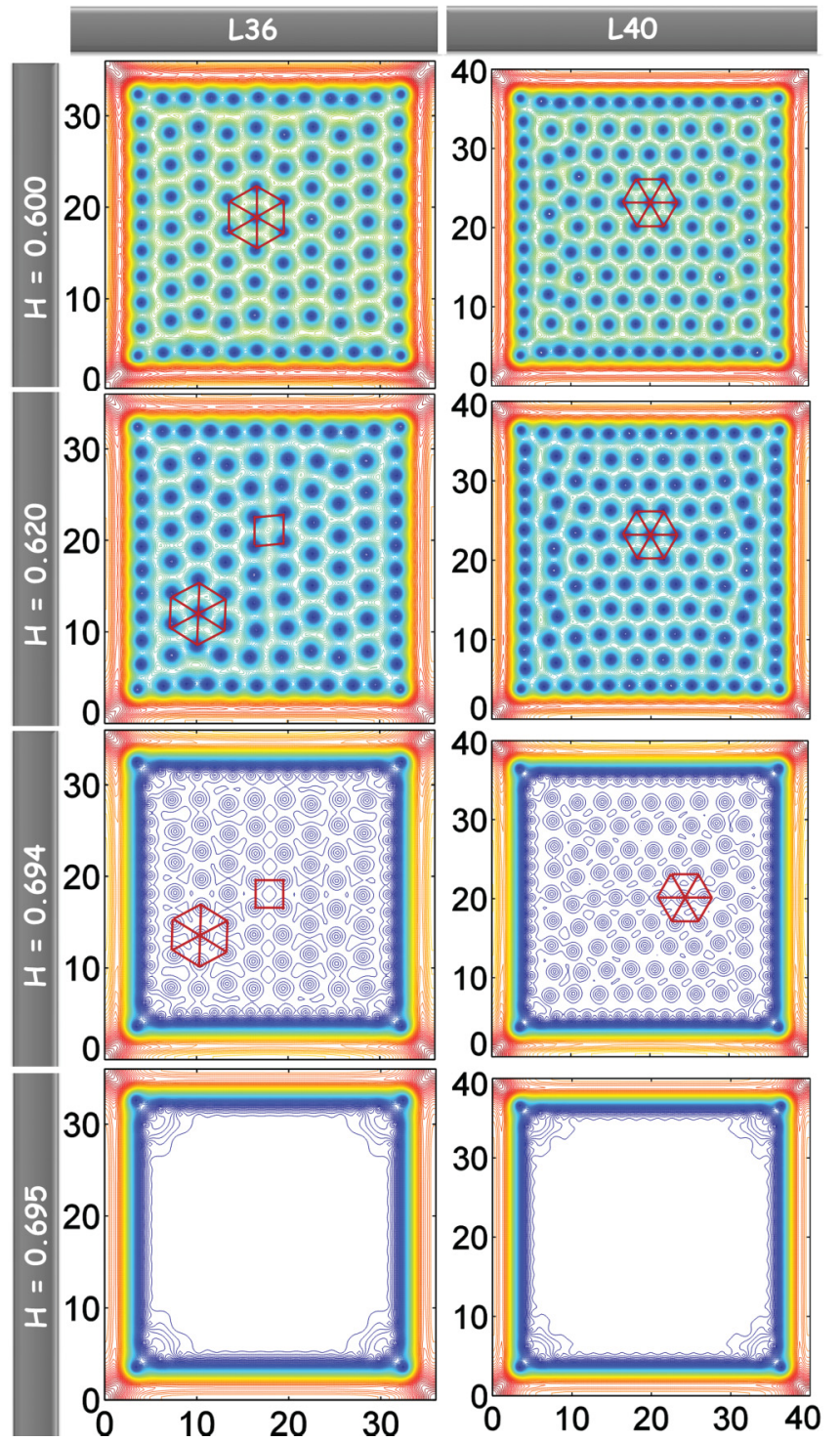

FIG. 5. (Color online) Vortex lattice for samples $L=36$ and $L=40$ for several values of the external field, at $T=0.3125$. Notice that, for the macro sample $(L=40)$, vortices always form a hexagonal lattice. A crossover between hexagonal and square lattices takes place for the mesoscopic sample $(L=36)$. Right below $L_{c}$, the system starts developing a hexagonal lattice for low densities of vortices, but still carries vestiges of the mesoscopic regime as this density increases, developing a mixture of square and hexagonal vortex lattice.

at $T=0.3125$ (Fig. 5) feature occurs for samples $L=36$ and $L=40$ for $H \geqslant 0.695$.

As stressed before, the vortex lattice always displays hexagonal symmetry for sample dimensions above $L_{c}$. A quantitative evidence, that the picture that comes out from the simulations indeed corresponds to a triangular lattice, can be obtained from the structure factor of the system. Figure 6 shows contour plots of the order parameter [Figs. 6(a) and 6(c)] and the corresponding structure factors of the vortex lattice for sample $L=128$ at $H=0.126$ [Fig. 6(b)] and $H=0.127$ [Fig. 6(d)]. Considering the peak values of the structure factor in Fig. 6(b), we obtained the vector basis oriented

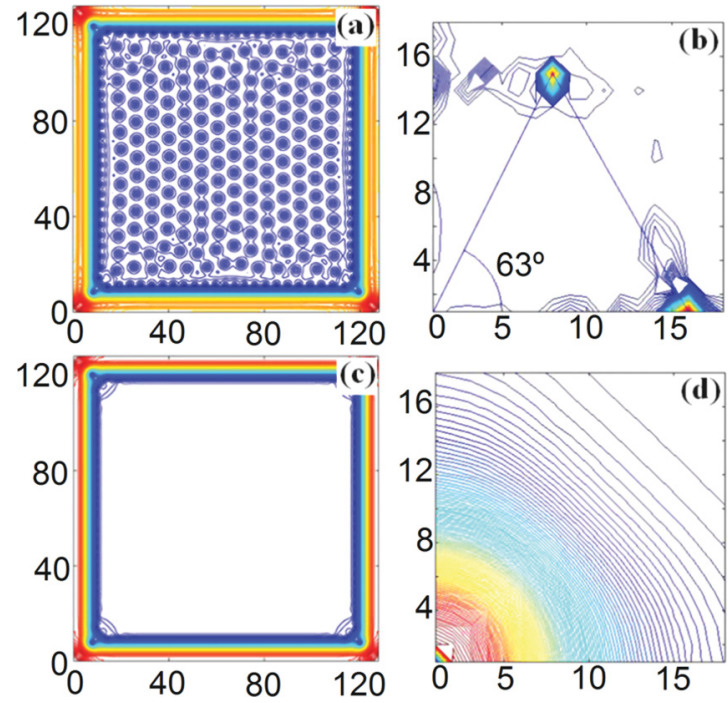

FIG. 6. (Color online) Topology of the superconducting density of the Cooper pairs $|\psi|^{2}$, and its corresponding structure factors, for sample $L=128$. Top: $H=0.126$, bottom: $H=0.127$. Notice that the vortex lattice disappears abruptly at $H=0.127$ [panels (c) and (d)], close to the Abrikosov bulk upper critical field $H_{c 2}(T=$ $0.875)=0.125$. The structure factor was calculated as the absolute value of the fast Fourier transform of $|\psi|$.

as approximately an equilateral triangle, as expected for an Abrikosov type-II superconductor. Notice that just a small increase in the applied field destroys the vortex lattice and the peaks in the structure factor disappear [Figs. 6(c) and 6(d)]. It is therefore clear that, for $L=128$, the system is already in the macroscopic regime, exhibiting a genuine Abrikosov lattice, which disappears along with volume superconductivity.

In order to construct an $L-T$ phase diagram, we repeated, for several temperatures, the procedure employed to determine $L_{c}$, as discussed above and exemplified in Figs. 1 and 2. The resulting phase diagram is shown in Fig. 7. Such diagram separates the region where the superconductor behaves predominantly as a macroscopic sample from that where a mesoscopic behavior takes place. We have used the expression $L_{c}(T)=L_{0}(1-T)^{n}$ to fit the data, considering $L_{0}$ and $n$ as free adjustable parameters. The value of $L_{0}$ corresponds to the length scale for which the mesoscopic-macroscopic transformation occurs at zero temperature; $n$ is the critical exponent. The best fit, shown in Fig. 7 along with the data, gives $L_{0}=36.5$ and $n=-0.292 \approx-2 / 7$. Thus, it is clear that the length scale for the crossover between both regimes is much larger than the London penetration depth $\lambda(T)=\kappa / \sqrt{1-T}$. A comparison between $L_{c}(T)$ and $\lambda(T)$ can be seen in Fig. 7. In real units, if we take $\xi(0)=10 \mathrm{~nm}$, which is a typical size of low $T_{c}$ type-II superconductors, then the value of $L_{c}(T)$ would range from 365 to $1150 \mathrm{~nm}$ as $T$ varies from zero to 0.9375 . This is of the same order of magnitude where size effects disappear in nanoparticle systems. ${ }^{31}$

The threshold line changes very little for different materials, as one can infer from the two curves shown in Fig. 7, for $\kappa=5$ and $\kappa=\infty$. Not only the values for the infinity $\kappa$ limit of the TDGL equations differ only a few percent from those obtained for $\kappa=5$, but also the overall tendency of the two curves is similar, to an extent that the fitting exponent for both 


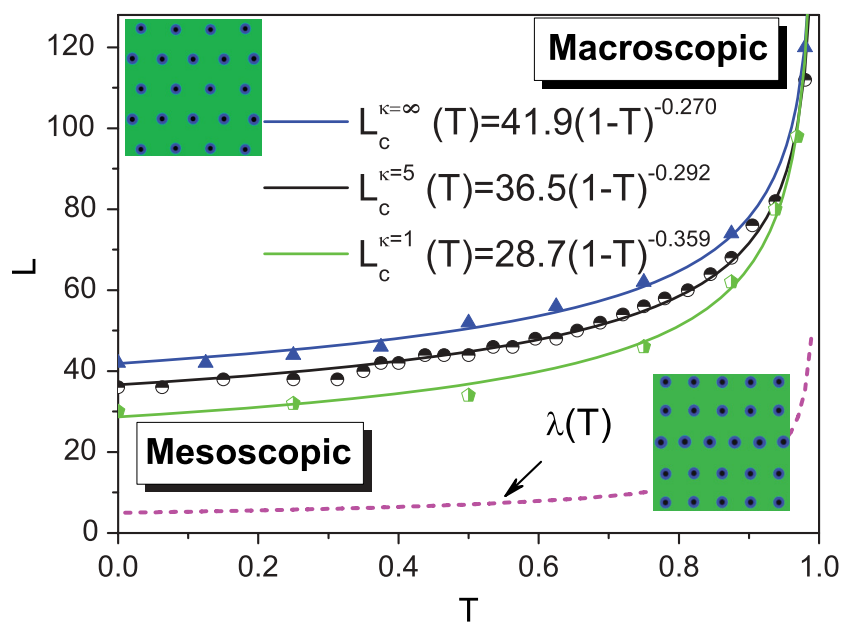

FIG. 7. (Color online) The $L_{c}-T$ phase diagram; above the critical line the superconductor behaves as a macroscopic sample; below it, a mesoscopic behavior takes place. The insets indicate that for $L>L_{c}$ the lattice is always hexagonal, whereas for $L<L_{c}$ the system may develop other types of symmetries (see text). $\lambda(T)$ for $\kappa=5$ is also shown for comparison.

is virtually the same $(\sim-2 / 7)$, as evidenced in Fig. 7. It is not surprising that there is no significant differences between the characteristics of the $L_{c}(T)$ curves, since the vortex states are indistinguishable for $\kappa \geqslant 5$, as it was demonstrated in Ref. 32. For low values of $\kappa$ the $L_{c}(T)$ curve presents the same dependence with $T$, however, if such curve is compared with that one for $\kappa=5$, significant changes can be observed in the value of the $n$ exponent, which is approximately $-5 / 14$, and the value of $L_{0}$.
Recently, Connolly et al. ${ }^{33}$ have made a similar study in order to find the meso-to-macro crossover for a superconducting disk. They used a criterion based in the competition between Abrikosov vortex lattice and shell-like ordering. As a result, they obtained that the crossover occurs in the diameter range of

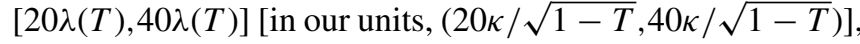
which differs from our results for large $\kappa$.

As a final remark, we can surely say that our mapping of the frontier between the mesoscopic and macroscopic length scale could be an important guide for the fabrication of samples with desirable dimensions for which one can observe mesoscopic effects in superconductivity.

\section{CONCLUSION}

In short, based on the physical evidence that, in the mesoscopic regime, vortices nucleating at the sample surface interact with each other even at the very early stages of their inception, we have developed a systematic procedure to determine the crossover between mesoscopic and macroscopic regimes of superconductivity in small samples. The progressive change of symmetry of the vortex lattice, from square to hexagonal, is intimately related to the meso-tomacro crossover and further emphasizes the robustness of our criterion.

\section{ACKNOWLEDGMENTS}

The authors thank the Brazilian agencies Fundação de Amparo à Pesquisa do Estado de São Paulo (FAPESP) and Conselho Nacional de Desenvolvimento Científico e Tecnológico (CNPq) for financial support. W.A.O. acknowledges the Centre for Advanced Study (Norway) for the hospitality during the last stage of this work.
${ }^{1}$ W. P. Halperin, Rev. Mod. Phys. 58, 533 (1986).

${ }^{2}$ Y. G. Sun and Y. N. Xia, Science 298, 2176 (2002).

${ }^{3}$ D. J. Harrison, K. Fluri, K. Seiler, Z. H. Fan, C. S. Effenhauser, and A. Manz, Science 261, 895 (1993).

${ }^{4}$ F. Baletto and R. Ferrando, Rev. Mod. Phys. 77, 371 (2005).

${ }^{5}$ A. K. Geim, S. V. Dubonos, J. G. S. Lok, M. Henini, and J. C. Maan, Nature (London) 396, 144 (1998).

${ }^{6}$ A. K. Geim, S. V. Dubonos, I. V. Grigorieva, K. S. Novoselov, F. M. Peeters, and V. A. Schweigert, Nature (London) 407, 55 (2000).

${ }^{7}$ M. Morelle, J. Bekaert, and V. V. Moshchalkov, Phys. Rev. B 70, 094503 (2004).

${ }^{8}$ D. S. Golubovic, M. V. Milošević, F. M. Peeters, and V. V. Moshchalkov, Phys. Rev. B 71, 180502 (2005).

${ }^{9}$ A. Kanda, B. J. Baelus, F. M. Peeters, K. Kadowaki, and Y. Ootuka, Phys. Rev. Lett. 93, 257002 (2004).

${ }^{10}$ B. J. Baelus, A. Kanda, F. M. Peeters, Y. Ootuka, and K. Kadowaki, Phys. Rev. B 71, 140502 (2005).

${ }^{11}$ M. V. Milošević, A. Kanda, S. Hatsumi, F. M. Peeters, and Y. Ootuka, Phys. Rev. Lett. 103, 217003 (2009).

${ }^{12}$ T. Cren, L. Serrier-Garcia, F. Debontridder, and D. Roditchev, Phys. Rev. Lett. 107, 097202 (2011).
${ }^{13}$ I. V. Grigorieva, W. Escoffier, V. R. Misko, B. J. Baelus, F. M. Peeters, L. Y. Vinnikov, and S. V. Dubonos, Phys. Rev. Lett. 99, 147003 (2007).

${ }^{14}$ S. Okayasu, T. Nishio, Y. Hata, J. Suzuki, I. Kakeya, K. Kadowaki, and V. V. Moshchalkov, IEEE Trans. Appl. Supercond. 15, 696 (2005).

${ }^{15}$ T. Nishio, Q. Chen, W. Gillijns, K. De Keyser, K. Vervaeke, and V. V. Moshchalkov, Phys. Rev. B 77, 012502 (2008).

${ }^{16}$ T. Nishio, S. Okayasu, J. Suzuki, and K. Kadowaki, Physica C 412, 379 (2004); 414, 379 (2004).

${ }^{17}$ L. F. Chibotaru, A. Ceulemans, V. Bruyndoncx, and V. V. Moshchalkov, Nature (London) 408, 833 (2000).

${ }^{18}$ V. A. Schweigert, F. M. Peeters, and P. S. Deo, Phys. Rev. Lett. 81, 2783 (1998).

${ }^{19}$ A. S. Mel'nikov, I. M. Nefedov, D. A. Ryzhov, I. A. Shereshevskii, V. M. Vinokur, and P. P. Vysheslavtsev, Phys. Rev. B 65, 140503 (2002).

${ }^{20}$ B. J. Baelus and F. M. Peeters, Phys. Rev. B 65, 104515 (2002).

${ }^{21}$ E. Sardella, A. L. Malvezzi, P. N. Lisboa-Filho, and W. A. Ortiz, Phys. Rev. B 74, 014512 (2006). 
${ }^{22}$ B. Xu, M. V. Milošević, S. H. Lin, F. M. Peeters, and B. Janko, Phys. Rev. Lett. 107, 057002 (2011).

${ }^{23}$ E. Sardella, P. N. Lisboa-Filho, and A. L. Malvezzi, Phys. Rev. B 77, 104508 (2008).

${ }^{24}$ L. F. Chibotaru, A. Ceulemans, V. Bruyndoncx, and V. V. Moshchalkov, Phys. Rev. Lett. 86, 1323 (2001).

${ }^{25}$ L. F. Chibotaru and V. V. Moshchalkov (private communication).

${ }^{26}$ A. Schmid, Phys. Kondens. Mater. 5, 302 (1966).

${ }^{27}$ R. Geurts, M. V. Milošević, and F. M. Peeters, Phys. Rev. B 81, 214514 (2010).
${ }^{28}$ W. D. Gropp, H. G. Kaper, G. K. Leaf, D. M. Levine, M. Palumbo, and V. M. Vinokur, J. Comput. Phys. 123, 254 (1996).

${ }^{29}$ M. V. Milošević and R. Geurts, Physica C 470, 791 (2010).

${ }^{30}$ Fourfold symmetry: first penetration involves simultaneous nucleation of four vortices.

${ }^{31}$ A. S. Barnard, Rep. Prog. Phys. 73, 1 (2010).

${ }^{32}$ Q. Du, M. D. Gunzburger, and J. S. Peterson, Phys. Rev. B 51, 16194 (1995).

${ }^{33}$ M. R. Connolly, M. V. Milošević, S. J. Bending, J. R. Clem, and T. Tamegai, Europhys. Lett. 85, 17008 (2009). 